\title{
Health Education Module for Stroke Caregivers
}

\author{
Waled Amen Mohammed Ahmed ${ }^{1 *}$, Ehab Ibrahim Salih El-Amin ${ }^{2}$, Ragaa G Ahmed ${ }^{1}$, Ziad M Alostaz ${ }^{1}$ and Khalid Eltahir Khalid ${ }^{3}$ \\ ${ }^{1}$ Department of Nursing, Faculty of Applied Medical Sciences, Albaha University, Kingdom of Saudi Arabia \\ ${ }^{2}$ Department of Community Health, Faculty of Applied Medical Sciences, Albaha University, Kingdom of Saudi Arabia \\ ${ }^{3}$ Department of Medical Laboratory Sciences, Faculty of Applied Medical Sciences, Albaha University, Kingdom of Saudi Arabia \\ "Corresponding author: Waled Amen Mohammed Ahmed, Department of Nursing, Faculty of Applied Medical Sciences, Albaha University, Kingdom of Saudi Arabia,
} Tel: 00966508245369; E-mail: weliameen1980@yahoo.com

Rec date: Aug 23, 2014, Acc date: Feb 16, 2015, Pub date: Feb 18, 2015

Copyright: (c) 2015 Ahmed WAM, et al. This is an open-access article distributed under the terms of the Creative Commons Attribution License, which permits unrestricted use, distribution, and reproduction in any medium, provided the original author and source are credited.

\begin{abstract}
Stroke is the third leading cause of death in world. According to the Centers for Disease Control and Prevention (CDC), the incidence is around 1 in every 40 seconds and the death rate is in every 4 minutes in USA. People under the age of 65 are prone to 255 of stroke attacks. Today, approximately 6.2 million people are living with the consequences of stroke. Incidence is nearly double that of Caucasians and African Americans suffer more extensive physical impairments. This module provides trainers with an outline for the teaching of the basic care for stroke patients. Participants will learn about stroke as a disease and its nature then basic care for stroke patients will be demonstrated.
\end{abstract}

Keywords: Stroke; Caregivers

\section{Introduction}

Stroke is the third leading cause of death in world. According to the Centers for Disease Control and Prevention (CDC), someone in the United States has a stroke every 40 seconds, and every 4 minutes someone dies of stroke. Although stroke risk increases with age, strokes can and do occur at any age. Nearly a quarter of strokes occur in people under the age of 65 . Today, approximately 6.2 million people are living with the consequences of stroke [1]. Incidence is nearly double that of Caucasians and African Americans suffer more extensive physical impairments.

While many people survive their first stroke, stroke survivors and their caregivers often don't have the information they need to manage their recovery process at home. A competent and knowledgeable caregiver is critical in determining whether a stroke survivor's mental and physical health improves, and whether the survivor or a stroke is institutionalized or can remain at home in his or her community. Because strokes occur suddenly, survivors and caregivers need extensive education to prevent second strokes and to manage the aftermath of stroke [1].

Education is a vital aspect of care and management of debilitating illnesses, especially stroke. In a presentation given at the $2012 \mathrm{New}$ Horizons in Rehabilitation Conference, a team of Veteran Affairs (VA) nurses evaluated two stroke education programs developed by $\mathrm{VA}$, one administered in person and one web-based, and found that both were important new tools in helping Veterans with stroke and their caregivers to manage the disease. More than one-third of caregivers suffer from poor health themselves. 29\% of female caregivers have passed up a job promotion, training or assignment to take care of a stroke-survivor. Respite and social support make caregivers less stressed, more satisfied, and better able to provide care for longer periods of time [2].
This module provides trainers with an outline for the teaching of the basic care for stroke patients. Participants will learn about stroke as a disease and its nature then basic care for stroke patients will be demonstrated.

\section{Methods}

The method of delivery for this module will be as follow; individual health education, lectures, home visits and posters distribution. The main intervention focused on a number of family caregivers. The process of evaluation based on ascertained changes of family caregivers' knowledge and behavior about care of stoke patients at home, whereas the outcome evaluation based on quality of care provided.

Participants will take two days training and listen to lectures on topics including understanding stroke, functional recovery, and communication and cognition. After the lecture, those in attendance will hold interactive discussions with the hospital's stroke team and also discuss appropriate support for each individual participant. After every session, evaluation forms will be passed out to attendees.

\section{Focus}

Throughout this module, the focus is on improving quality of stroke caregivers' skills

\section{Aim}

The primary aim is to improve the knowledge of stroke caregivers about daily and regular home care. The secondary aim is to improve the quality of care.

\section{Objectives}

- Identify underlying causes of different types of stroke. 
- Learn about basic measures to promote regular and daily care of stroke patients.

- Demonstrate the ability to systematically evaluate condition of stroke patients.

- Demonstrate specific preventive measures for bedsores.

- Educate others about prevention of bedsores.

- Learn basic skills needed for stroke patients (personal hygiene, bathing, bed care, transferring, wheeling-wheel chairs, special clothing needs (dressing), medication organization, aphasia, some help hints for dealing with aphasia, subluxation dislocated shoulder, skin breakdown, loss of bladder-bowel functions, feeding.)

\section{Participants}

This module is intended for the stroke caregivers, who wish to improve their knowledge and skills in care of stroke patients and thus improve the quality of care. It is expected that the family caregivers using this module will have foundation knowledge in providing basic needs and preventive strategies of bedridden complications and skills of immediate intervention using available and affordable resources.

\section{Expected Outcome of this Module}

- Enhance stroke caregivers' awareness to promote a safe care.

- Provide basic needs such as personal hygiene, bathing, bed care, transferring, wheeling-wheel chairs, special clothing needs (dressing), medication organization, aphasia, some help hints for dealing with aphasia, subluxation dislocated shoulder, skin breakdown, loss of bladder-bowel functions, feeding.

- Build caregivers' skills in providing basic needs, and immediate intervention especially among bedridden patients.

- Administer basic immediate interventions in the absence of medical help to reduce the severity of episodes.

- Also it is expected to reduce mortality and morbidity of stroke patients by providing immediate interventions through minimizing complications.

\section{How to Use this Module}

This module has been designed by collecting pictures and information to illustrate the skills needed for providing basic daily needs of stroke patients and basic skills for prevention of bedsores and demonstrate immediate interventions for urgent events thus, because of suspected illiteracy among family caregivers.

The module includes a set of simplified lectures, posters that will improve the general understanding of stroke and help to remember the preventive measures and basic daily needs. If the family caregivers unable to complete an action we will encourage him/her to re-observe the pictures that precedes the action and then attempt the action again. It is important to understand each picture as you work through the module to build on those that preceded it. Sufficient informational pictures will be included in the module to allow for its understanding.

\section{Primary Messages}

- Stroke is happen suddenly.

- There are two main types of stroke (heamorrhagical and nonheamorrhagical).
- To prevent bedsores, it is important to understand and anticipate their causes.

- Stroke patients needs help in their daily living activities.

- Bathing, hygiene, feeding, moving and turning activities take top priorities.

- Stroke patients needs home care skills such as personal hygiene, bathing, bed care, transferring, wheeling-wheel chairs, special clothing needs (dressing), medication organization, some help hints for dealing with aphasia, subluxation dislocated shoulder, skin breakdown, loss of bladder-bowel functions, feeding

- Family caregivers for stroke patients need training to ensure providing a good quality of care (Figure 1).

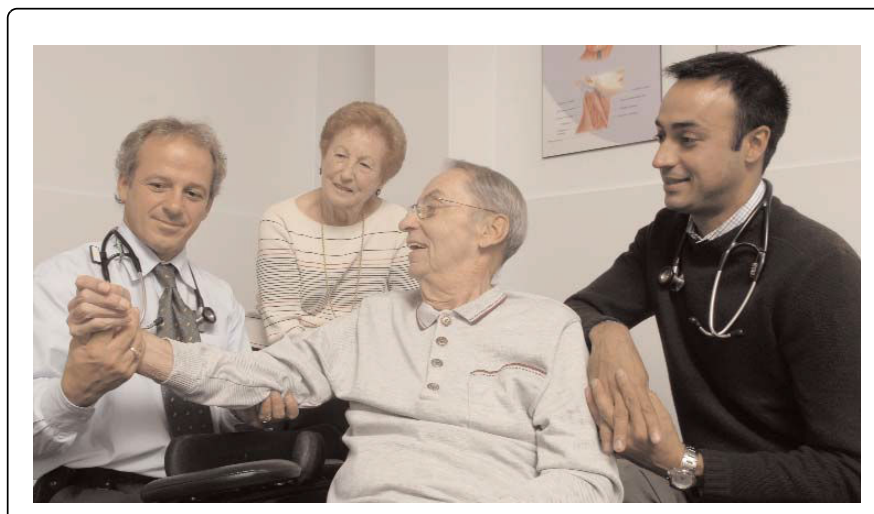

Figure 1: Family caregivers for stroke patients need training to ensure providing a good quality of care.

\section{Principles for Stroke Caregivers}

- Make time for yourself. You will be a better caregiver if you take time to do things you enjoy.

- Set priorities. Identify what is most important. Say "no" to things that are less important.

- Accept that you cannot do everything. Don't feel guilty about asking for help.

- Have a "back-up" plan. Know who will provide care during emergencies.

- Find ways to make your life easier. You may need to hire someone to do chores.

- Be creative [3].

\section{Basic Care Sessions for Stroke Caregivers}

\section{Contents}

\section{A. Overview of stroke}

\section{1 . What is stroke?}

2. Characteristics of the stroke

3. Symptoms of stroke

4. Risk factors

5. Treatment of the stroke

6. Prevention of complications 


\section{B. Training sessions for stroke caregivers}

1. Personal hygiene

2. Bathing

3. Bed care

4. Transferring

5. Wheeling-wheel chairs

6. Special clothing needs (Dressing)

7. Medication organization

8. Aphasia

9. Some help hints for dealing with aphasia

10. Subluxation dislocated shoulder

11. Skin breakdown

12. Loss of bladder-bowel functions

13. Feeding

\section{Overview of stroke}

Stroke has no regards or respect for age, race, creed, color, intelligence, accomplishments. You and those around you may not even be aware of what is happening nor know what to do in this most critical of times. You may have a warning in the form of all strokes are caused by an interruption of blood to the brain, so there may be symptoms in common, but since everyone's brain is different, so is practically every stroke. The blood supply to the brain is disrupted or diminished, and that situation comes about because of:

1. Blockage (a "block")

2. Bleeding (a "bleed")

The "block" can be a blood clot, fatty material or any foreign matter getting stuck in an artery like debris-laden water through a clogged pipe: at most, only a trickle of blood can get through, and the brain needs more than a trickle to deliver the required amount of oxygen to function properly. These strokes can also be called thrombotic (cerebral thrombosis, a clot formed at the site of blockage), embolic (embolism, a clot, air or foreign object formed elsewhere that travels to the site of blockage) or ischemic (a catchall word for any type of blockage).

There is also some evidence, though rare, that vessels can spasm or contract and shut off blood flow, causing a blockage of blood to the brain. A "bleed" occurs when a blood vessel bursts, allowing blood to flow into the brain or its surrounding area. Other terms associated with bleeds are haemorrhage and ruptured aneurysm.

When the new stroke victim is in the hospital, probably first in intensive care and then in regular care, that is when the designated caregiver has to step to, every hospital patient needs an advocate to help look out for their well-being. A stroke victim, because they have suffered an attack to the brain, may have more than the usual amount of difficulty with communication, comprehension and of course, mobility.

\section{Things you should know about stroke}

- One in five people having a first-ever stroke die within one month and one in three die within a year.
- The number of strokes will increase each year due to the ageing population unless something is done to reduce the incidence rate.

- The FAST test is an easy way to recognize and remember the signs of stroke. Using the FAST test involves asking three simple questions:

Face-Check their face. Has their mouth drooped?

Arms-Can they lift both arms?

Speech-Is their speech slurred? Do they understand you?

Time-Time is critical. If you see any, take the victim to the nearest health setting.

- In the next 10 years, more than half a million people will suffer a stroke.

- Stroke kills more women than breast cancer and more men than prostate cancer.

- About 88 percent of stroke survivors live at home and most have a disability.

- Close to 20 per cent of all strokes occur to people under 55 years old.

\section{What is a stroke?}

1. Syndrome caused by disruption of blood flow to part of the brain.

2. Occurs when blood vessel either ruptures or becomes occluded.

3. Deprives neurons and other brain cells of glucose and oxygen leading to cell death.

\section{Results in impaired or lost function.}

\section{Characteristics of stroke}

- Acute onset of one or more neurological deficits that:

- Begin abruptly

- Persist for at least $24 \mathrm{hrs}$

- Reflect focal involvement of central nervous system Ischemic Stroke

- Diplopia

- Vertigo

- Coma at onset

- Crossed sensory loss

- Bilateral motor signs

- Isolated field defect

- Pure motor and sensory deficit

- Dysarthria

- Dysphagia

\section{Symptoms of a stroke can include}

- A numb or weak feeling in the face, arm or leg.

- Trouble of speaking or understanding.

- Unexplained dizziness.

- Blurred or poor vision in one or both eyes.

- Loss of balance or an unexplained fall.

- Difficulty swallowing.

- Headache (usually severe or of abrupt onset) or unexplained change in the pattern of headaches confusion.

\section{Risk factors of stroke}

- Strong family history of stroke

- Heavy consumption of alcohols \& addict drugs

- Obesity 
- Heavy smoking

- High blood pressure

- Diabetes mellitus

- Vascular disorders

- Atrial fibrillation

- High lipid profile

\section{Treatments of stroke}

Every patient should be treated according to his condition because there is variation in the aetiolgy of stroke so every case should be treated individually but the important thing is to take drugs as ordered.

\section{Prevention of complications}

Staff should position patients, whether lying or sitting, to minimize the risk of complications such as aspiration, respiratory complications, shoulder pain, contractures and pressure sores.

Compression stockings should be applied in stroke patients with weak or paralysed legs (once the patient's peripheral circulation, sensation and the state of the skin have been assessed).

\section{Care training session for stroke caregivers}

\section{Personal hygiene}

Mouth care: to keep mouth clean free from flora brushing or use tongue depressor to demonstrate cleaning with normal saline.

\section{Bathing}

Cotton towels get heavy when wet. Switch to hand towels for drying or lighter weight bath towels. Bathing sponges on a stick are useful for scrubbing feet, the back and other hard to reach places.

If the survivor is unable to use the bath, then there are alternative ways for bathing and toileting. A sponge bath, If they cannot be moved from the bed, roll them over, place a plastic sheet and towels on the bed and roll them back. You can use a basin of warm water to give a modified wet bath.

\section{Bathroom safety features}

Bathrooms are potentially hazardous places because of slippery hard surfaces.

The bathroom can pose its own challenges, as well as its own hazards due to slippery or hard surface areas. A handheld shower will enable the survivor to bathe himself if they have the use of one.

\section{Bed care}

If possible, clear the bedside area of rugs. They may be prone to accidental spills and urine odor is difficult to get rid of. A vinyl mattress protector will safeguard against accidents.

Clothing and linens soiled with body waste should be washed separately in hot water and detergent. If the clothing is heavily soiled, it may need to be put through the wash cycle twice if available.

\section{Transferring}

Transfers to and from the wheelchair and toilet need to be practiced. The main concern is preventing the survivor from falling and the family caregiver from putting their back out. If the survivor is right side affected from the stroke, always transfer them to their left (strong) side. When getting them out of bed, park the wheelchair so that they can reach across with their left hand and pull themselves from the bed to the chair with minimal assistance from the caregiver. Obviously, the opposite instructions apply to somebody whose left side has been affected by stroke

\section{Wheeling wheelchairs}

Extra furniture or lamps may need to be removed to widen the wheelchair opening. If turning areas are tight and the wheelchair will bump into cabinets, door moldings and the like, corners can be protected with bump guards either purchased or homemade.

\section{Special clothing needs (dressing)}

Research has found that one of the biggest problems amongst stroke survivors is dressing themselves. If the stroke survivor has been left with paralysis or weakness on one side, garments may need to be replaced with ones that take these limitations into consideration. Even if there is no hemi paresis, buttons, zippers, hooks and other fasteners should be examined for ease of use.

Trousers-Elastic waistbands or a partial elastic waist are preferable. Pants with Velcro closures, and other features that make dressing easier for the survivor. Zippers should slide easily and have large enough tabs or tab attachments that make it easier to grasp.

Shirts-Traditional men's shirts generally have small buttons and buttons on the cuff that may be hard to deal with. If possible, switch to washable knit shirts as they may be easier to work with.

Underwear-It should be comfortable, not tight or binding.

\section{Aphasia}

Most individuals with aphasia are competent mentally and have very frustrating disorder. They know what they want to say; they just have trouble putting their thoughts into speech or writing.

The good news is that aphasia doesn't get worse unless there's another stroke or brain injury. Almost all people improve. How much and when it will happen falls into the "each survivor is different" category. The primary goal is to learn to cope with the problem and communicate effectively despite the aphasia.

\section{To reach this goal, some helpful hints for dealing with aphasia}

1. A call bell, bicycle horn or other noisemaker can be used by the survivor to signal that something requires attention.

2. Basic letter boards and picture boards help the patient express. Elementary school flash cards can be used in a variety of ways.

3. Index cards can communicate most commonly needed items and expressions:

Bedpan, Water, Thank you, Sorry, I love you, Help.

4. Laminate them to last longer. Labels on all objects in the room that they may want to use: telephone, lamp, TV, glass, book. Name everything you touch as you use it. Aphasics frequently lose the names of objects or numbers. They may call everything a "key" or person's name and although they know dinner is a six, they may call it "ten o'clock."

5. Speak slowly and clearly (not loudly, they can still hear), one person at a time. Being aphasic in a roomful of people is like having that many televisions all set to different channels at the same time. It's impossible, confusing and upsetting to follow any one plot. 
Page 5 of 5

6. Assume the patient can understand and don't say anything negative, or what you wouldn't want them to hear, in front of them.

7. Encourage the patient to talk even if the words aren't correct or are just repeating what you say. Not talking for fear of embarrassment is worse than trying and not doing it well. Try not to answer for the individual, as practice is necessary for improvement.

\section{Skin breakdown}

Protect skin from breaking down by moving the patient often, keeping the skin clean, aerated and dry, and having any red spots or sores treated as soon as they appear. The use of a bedpan frequently may cause skin irritation. Adult diapers may be a better idea.

\section{Loss of bladder/Bowel functions}

Often after a stroke, survivors find it difficult to pass urine or are unable to control when the urine is passed, and the same is often true of bowel movements. While this could be caused by damage to the part of the brain that governs control, it may not be a problem of incontinence, but a problem of communication. The patient may be unable to communicate the need to empty their bladder. It is important that the family caregiver be sensitive to this situation.

Depression/Emotional issues
Stroke, like death, represents a catastrophic personal loss. The only way to heal, to be able to cope, is through a grieving process of several stages. There is (in no particular order) shock, anger, relief, depression, denial, mourning, acceptance, and a moving on. Grief is a very personal experience that takes time.

Feeding: Most of the stroke patients discharged from the hospital after two weeks without (NGT) nasogastric tube as are dependent on feeding by oral method so the family caregiver is aware of nutritional needs of his relative regarding the food contain all essential nutrients [4].

\section{References}

1. Ministry of Health and Long Term Care and the Heart and Stroke Foundation of Ontario, Towards an Integrated Stroke Strategy for Ontario: Report of the Joint Stroke Strategy Working Group (2000).

2. Cheung D, McKellar J, Bereket T (2010) Collaborative Interprofessional Stroke Care in Community Re-engagement. Toronto West and South East: Ontario Stroke system.

3. Resources and Education for Stroke Caregivers' Understanding and Empowerment (2012).

4. Stroke caregiver-Handbook (2008) In: Members of Stroke Network in USA (ed.) New York. 